\title{
Impairment of Pharyngoesophageal Segment Opening
}

National Cancer Institute

\section{Source}

National Cancer Institute. Impairment of Pharyngoesophageal Segment Opening. NCI

Thesaurus. Code C127319.

An observation of an individual's pharyngoesophageal seg ment opening during swallowing. 